$11-1-2012$

\title{
On Some Properties and Estimation of Size-Biased Polya-Eggenberger Distribution
}

Anwar Hassan

King Saud University, Riyadh, Kingdom of Saudi Arabia

Sheikh Bilal

A. S. College, Srinagar, Kashmir, India

Imtiyaz Ahmad Shah

Indian Institute of Technology-Dehli, New Dehli, india

Follow this and additional works at: http://digitalcommons.wayne.edu/jmasm

Part of the Applied Statistics Commons, Social and Behavioral Sciences Commons, and the Statistical Theory Commons

\section{Recommended Citation}

Hassan, Anwar; Bilal, Sheikh; and Shah, Imtiyaz Ahmad (2012) "On Some Properties and Estimation of Size-Biased PolyaEggenberger Distribution," Journal of Modern Applied Statistical Methods: Vol. 11 : Iss. 2 , Article 10.

DOI: $10.22237 /$ jmasm/1351742940

Available at: http://digitalcommons.wayne.edu/jmasm/vol11/iss2/10

This Regular Article is brought to you for free and open access by the Open Access Journals at DigitalCommons@WayneState. It has been accepted for inclusion in Journal of Modern Applied Statistical Methods by an authorized editor of DigitalCommons@WayneState. 


\section{On Some Properties and Estimation of Size-Biased Polya-Eggenberger Distribution}

\section{Cover Page Footnote}

This project was supported by the Research Center, College of Science, King Saud University, Riyadh, Saudi Arabia. 


\section{On Some Properties and Estimation of Size-Biased Polya-Eggenberger Distribution}

\author{
Anwar Hassan \\ King Saud University \\ Riyadh, Kingdom of Saudi Arabia
}

\author{
Sheikh Bilal \\ A. S. College, Srinagar \\ Kashmir, India
}

Imtiyaz Ahmad Shah

Indian Institute of Technology-Delhi

New-Delhi, India

A size-biased version of Polya-Eggenberger distribution is introduced explicitly and by a mixture model. The proposed distribution is unimodal with positive integer moments. The recurrence relation between moments (about the origin) of the proposed distribution is established and its relationship with other distributions is discussed. Different estimation techniques are proposed to estimate the parameters of the distribution.

Key words: Polya-Eggenberger distribution, size-biased Polya-Eggenberger distribution, recurrence relation between moments, estimation techniques.

\section{Introduction}

The Polya-Eggenberger distribution (PED), introduced by Polya and Eggenberger (1923) through an urn model and further analyzed by Polya (1930), is a discrete frequency distribution that was originally considered in connection with contagious distributions. The genesis of this distribution is expressed in terms of random drawings of colored balls from an urn: Initially, it is supposed that there are $a$ white balls and $b$ black balls in the urn; one ball is drawn at random and then replaced together with $s$ balls of the same color. If this procedure is repeated $\mathrm{n}$ times and $x$ represents the total number of times a white ball is drawn, then the distribution of $x$ is given by:

Anwar Hassan is an Associate Professor in the Department of Statistics \& Operations Research. Email him at: anwar.hassan2007@gmail.com or amohammadabdulkhair@ksu.edu.sa. Sheikh Bilal is a Senior Assistant Professor in the Department of Statistics. Email him at sbilal_sbilal@yahoo.com. Imtiyaz Ahmad Shah is a Research Scholar in the Department of Statistics and Operation Research. Email him at: imtiyaz3880@yahoo.co.in.

$$
\begin{aligned}
& P(x=k)= \\
& \left(\begin{array}{l}
n \\
x
\end{array}\right) \frac{a(a+s) \ldots .(a+\overline{x-1} s) b(b+s) \ldots .(b+\overline{n-x-1} s)}{(a+b)(a+b+s) \ldots .(a+b+\overline{n-1} s)}
\end{aligned}
$$

Where $x=0,1,2, \ldots, n$ and $n, a, b$ and $s$ are parameters of the distribution. The distribution is known as the Polya-Eggenberger distribution with parameters $(n, a, b, s)$.

Taking $\alpha=(a / s) ; \gamma=(b / s)$, results in an alternative form of (1.1) in ascending factorials as:

$$
\begin{aligned}
& P(x=k)=\left(\begin{array}{l}
n \\
k
\end{array}\right) \frac{\alpha^{[x]} \gamma^{[n-x]}}{(\alpha+\gamma)^{[n]}}, \\
& x=0,1,2, \ldots, n
\end{aligned}
$$

Distribution (1.2) is the most convenient form of PED for computational purposes. Another way to represent (1.1) is

$$
P(X=x)=\frac{\left(\begin{array}{c}
-a / s \\
x
\end{array}\right)\left(\begin{array}{c}
-b / s \\
x
\end{array}\right)}{\left(\begin{array}{c}
-(a+b) / s \\
x
\end{array}\right)}
$$$$
x=0,1,2, \ldots, n,
$$ 
and an alternative form of (1.1) in terms of parameters $\quad n ; \quad P=a /(a+b)$; $Q=1-P=b /(a+b)$ and $\delta=s /(a+b)$ is

$$
\begin{aligned}
& P(x=x)=\left(\begin{array}{l}
n \\
x
\end{array}\right) \frac{\prod_{j=0}^{x-1}(P+j \delta) \prod_{j=0}^{n-x-1}(Q+j \delta)}{\prod_{j=0}^{n-1}(1+j \delta)}, \\
& x=0,1,2, \ldots, n .
\end{aligned}
$$

It is possible for $s$ (and therefore $\delta$ ) to be negative, however $s$ must satisfy the inequality $(a+b)+s(n-1)>0$.

Srodka (1964) gave the recurrence relation among the moments about zero of the Polya-Eggenberger distribution (1.3) as:

$\mu_{r+1}^{\prime}=$

$(c+b+r s)^{-1} \sum_{j=0}^{r}\left[\begin{array}{c}a n\left(\begin{array}{c}r \\ j\end{array}\right)-(a-s n)\left(\begin{array}{c}r \\ j+1\end{array}\right) \\ -s\left(\begin{array}{c}r \\ j+2\end{array}\right)\end{array}\right] \mu_{r-j}^{\prime}$.

$r=0,1,2, \ldots$

The $r^{\text {th }}$ factorial moment is given by

$\mu_{(r)}=$

$n^{(r)} \prod_{j=0}^{r-1}\left[\frac{p+j \delta}{1+j \delta}\right] \sum_{k-r=0}^{n-r}\left(\begin{array}{l}n-r \\ k-r\end{array}\right) \frac{\left[\begin{array}{l}\prod_{j=0}^{k-r-1}\left(P^{\prime}+j \delta^{\prime}\right) \\ \times \prod_{j=0}^{n-k-1}\left(Q^{\prime}+j \delta^{\prime}\right)\end{array}\right]}{\prod_{j=0}^{n-r-1}\left(1+j \delta^{\prime}\right)}$

where

$$
\begin{gathered}
P^{\prime}=(P+r \delta)(1+r \delta)^{-1} \\
Q^{\prime}=Q(1+r \delta)^{-1}
\end{gathered}
$$

and

$$
\delta^{\prime}=\delta(1+r \delta)^{-1}
$$

Specifically, the first four central moments of the Polya-Eggenberger distribution are

$$
\begin{gathered}
\mu_{1}^{\prime}=n P=\frac{n a}{a+b} \\
\mu_{2}=n P Q(1+n \delta)(1+\delta)^{-1} \\
\mu_{3}=n P Q(Q-P)(1+n \delta)(1+2 n \delta)(1+\delta)^{-1}(1+2 \delta)^{-1}
\end{gathered}
$$

and

$$
\mu_{4}=\frac{n P Q(1+n \delta)\left[\begin{array}{c}
(1+2 n \delta)(1+3 n \delta)(1-3 P Q) \\
+(n-1)\{\delta+3 P Q(1+n \delta)\}
\end{array}\right]}{(1+\delta)(1+2 \delta)(1+3 \delta)}
$$

Models of Size-Biased Polya-Eggenberger Distribution (SBPED)

Size-biased distributions are a special case of the more general form known as weighted distributions. First introduced by Fisher (1934) to model ascertainment bias, weighted distributions were later formalized in a unifying theory by Rao (1965). Such distributions occur naturally in practice when observations from a sample are recorded with unequal probability, such as from probability proportional to size (PPS) designs. Briefly, if a random variable $\mathrm{X}$ has distribution $f(x, \theta)$, with unknown parameters $\theta$, then the corresponding weighted distribution is of the form

$$
f^{w}(x, \theta)=\frac{w(x) f(x, \theta)}{E[w(x)]}
$$

where $w(x)$ is a non-negative weight function such that $E[w(x)]$ exists.

A special case arises when the weight function is of the form $w(x)=x^{\beta}$. Such distributions are known as size-biased distributions of order $\beta$ (Patil\&Ord, 1976; Patil, 1981; Mahfoud\&Patil, 1982) and are written as: 


\section{SIZE-BIASED POLYA-EGGENBERGER DISTRIBUTION}

$$
f^{*}{ }_{\beta}(x, \theta)=\frac{x^{\beta} f(x, \theta)}{\mu_{\beta}^{\prime}}
$$

where $\mu_{\beta}^{\prime}=\sum_{x} x^{\beta} f(x, \theta)$ is the $\beta^{h}$ raw moment of $f(x, \theta)$.

If $X$ is a Polya-Eggenberger variate with a probability mass function as given by (1.2), then its mean is given by (1.6). The sizebiased version of $X$, known as the size-biased Polya-Eggenberger distribution (SBPED), can be obtained directly by taking $\beta=1$ in (2.2) and using (1.6). The resulting equation is:

$$
p(x)=\frac{x}{\frac{n \alpha}{(\alpha+\gamma)}}\left({ }_{x}^{n}\right) \frac{\alpha^{[x]} \gamma^{[n-x]}}{(\alpha+\gamma)^{[n]}}
$$

which can be put into the form:

$$
\begin{aligned}
& p(x)=\left(\begin{array}{c}
n-1 \\
x-1
\end{array}\right) \frac{(\alpha+1)^{[x-1]} \gamma^{[n-x]}}{(\alpha+\gamma+1)^{[n-1]},}, \\
& x=1,2, \ldots, n .
\end{aligned}
$$

Equation (2.3) gives the probability mass function (PMF) of the size-biased PolyaEggenberger distribution (SBPED).

\section{The Mixture Model}

The size-biased Polya-Eggenberger distribution can also be regarded as a beta mixture of size-biased binomial distribution. The PMF of the size-biased binomial distribution is given by

$$
\begin{aligned}
& P(x)=\left(\begin{array}{c}
n-1 \\
x-1
\end{array}\right) p^{x-1}(1-p)^{n-x}, \\
& 0<p<1, x=1,2, \ldots, n
\end{aligned}
$$

and the PDF of the beta distribution of first kind is

$$
\begin{aligned}
& f(p)=\frac{1}{\beta(\alpha, \gamma)} p^{\alpha-1}(1-p)^{\gamma-1}, \\
& 0<p<1 .
\end{aligned}
$$

Compounding (2.4) with (2.5) through the values of $p$ results in

$$
\begin{aligned}
P(x) & =\left(\begin{array}{c}
n-1 \\
x-1
\end{array}\right) \frac{1}{\beta(\alpha, \gamma)} \int_{0}^{1} p^{\alpha+x-2}(1-p)^{\gamma+n-x-1} d p \\
& =\left(\begin{array}{c}
n-1 \\
x-1
\end{array}\right) \frac{\beta(\alpha+x-1, \gamma+n-x)}{\beta(\alpha, \gamma)}
\end{aligned}
$$

which, after simplification, gives:

$$
\begin{aligned}
& p(x)=\left(\begin{array}{c}
n-1 \\
x-1
\end{array}\right) \frac{\alpha^{[x]} \gamma^{[n-x]}}{(\alpha+\gamma)^{[n-1]}}, \\
& x=1,2, \ldots, n .
\end{aligned}
$$

Replacing $\alpha$ with $\alpha+1$, (2.6) coincides with (2.3) and the PMF of the size-biased PolyaEggenberger distribution can be obtained.

Structural Properties of SBPED: Unimodality

The proposed model (2.3) is unimodal according to the results of Holgate (1970).

Lemma: If the mixing distribution is non-negative, continuous and unimodal, then the resulting distribution is unimodal.

The proposed model (2.3) is unimodal because the mixing distribution is a beta distribution, which is unimodal for $\alpha>1$ and $\gamma>1$.

Structural Properties of SBPED: Recurrence Relation between Probabilities

Taking $x=x+1$ in (2.3) and dividing the resulting equation by (2.3), results in the ratio

$$
\frac{P(x+1)}{p(x)}=\frac{(n-x)}{x} \frac{(\alpha+x)}{(\gamma+n-x-1)}
$$

which gives the recurrence relation between probabilities as:

$$
P(x+1)=\left[\frac{(n-x)}{x} \frac{(\alpha+x)}{(\gamma+n-x-1)}\right] p(x) .
$$


HASSAN, BILAL \& IMTIYAZ

Structural Properties of SBPED: Recurrence Relation between Moments

Multiplying (3.1) by $x^{k}$ and summing the resulting equation over $x$ results in

$$
\begin{aligned}
& \mu_{k+1}^{\prime}(n, \alpha, \gamma) \\
& =\sum_{x=1}^{n} x^{k} \frac{(n-1) !}{(n-x-1) !(x-1) !} \frac{(\alpha+1)^{[x]} \gamma^{[n-x]}}{(\alpha+\gamma+1)^{[n-1]}} \\
& =\frac{(n-1)(\alpha+1)}{(\alpha+\gamma+1)}\left[\begin{array}{r}
\sum_{x=1}^{n-1} x^{k} \frac{(\overline{n-1}-1) !}{(\overline{n-1}-x) !(x-1) !} \\
\times \frac{(\alpha+2)^{[x-1]} \gamma^{[\overline{n-1}-x]}}{(\alpha+\gamma+2)^{[\overline{n-1}-1]}}
\end{array}\right]
\end{aligned}
$$

which reduces to

$$
\mu_{k+1}^{\prime}(n, \alpha, \gamma)=\frac{(n-1)(\alpha+1)}{(\alpha+\gamma+1)} \mu_{k+1}^{\prime}(n-1, \alpha+1, \gamma)
$$

Equation (3.3) represents the recurrence relation between moments of proposed model (2.3). In particular

$$
\begin{gathered}
\mu_{1}^{\prime}=\frac{(n-1)(\alpha+1)}{(\alpha+\gamma+1)} \\
\mu_{2}^{\prime}=\frac{(n-1)(n-2)(\alpha+1)(\alpha+2)}{(\alpha+\gamma+1)(\alpha+\gamma+2)} \\
\mu_{3}^{\prime}= \\
\frac{(n-1)(n-2)(n-3)(\alpha+1)(\alpha+2)(\alpha+3)}{(\alpha+\gamma+1)(\alpha+\gamma+2)(\alpha+\gamma+3)}
\end{gathered}
$$

and

$\mu_{4}^{\prime}=$

$\frac{(n-1)(n-2)(n-3)(n-4)(\alpha+1)(\alpha+2)(\alpha+3)(\alpha+4)}{(\alpha+\gamma+1)(\alpha+\gamma+2)(\alpha+\gamma+3)(n-3)(\alpha+\gamma+4)}$
Structural Properties of SBPED: Factorial Moments

Suppose $\mu_{(k)}^{\prime}(n, \alpha, \gamma)$ denotes the $k^{t h}$ factorial moments of proposed model (2.3), then by definition

$$
\begin{aligned}
\mu_{(k)}^{\prime} & (n, \alpha, \gamma) \\
& =\sum_{x=1}^{n} x^{(k)} \frac{(n-1) !}{(n-x) !(x-1) !} \frac{(\alpha+1)^{[x]} \gamma^{[n-x]}}{(\alpha+\gamma+1)^{[n-1]}} \\
& =\sum_{x=k}^{n} x \frac{(n-1) !}{(n-x) !(x-k) !} \frac{(\alpha+1)^{[x-1]} \gamma^{[n-x]}}{(\alpha+\gamma+1)^{[n-1]}}
\end{aligned}
$$

Taking $x=x+k-1$ in (3.5) results in

$$
\begin{aligned}
& \mu_{(k)}^{\prime}=\sum_{x=1}^{n-k+1}(x+k-1) \\
& \frac{(n-1) !}{(\overline{n-k+1}-x) !(x-1) !} \frac{(\alpha+1)^{[x+k-2]} \gamma^{[\overline{n-k+1}-x]}}{(\alpha+\gamma+1)^{[n-1]}}
\end{aligned}
$$

and, after simplifying, gives:

$$
\begin{aligned}
& \mu_{(k)}^{\prime}=\frac{(n-1)^{(k-1)}(\alpha+1)^{[k-1]}}{(\alpha+\gamma+1)^{[k-1]}} \\
& \sum_{x=1}^{n-k+1}\left[\begin{array}{l}
(x+k-1) \times \frac{(\overline{n-k+1}-1) !}{(\overline{n-k+1}-x) !(x-1) !} \\
\times \frac{(\overline{\alpha+k-1}+1)^{[x-1]} \gamma^{[\overline{n-k+1}-x]}}{(\overline{\alpha+k-1}+\gamma+1)^{[\overline{n-k+1}-1]}}
\end{array}\right]
\end{aligned}
$$

This equation, together with (3.2), gives

$$
\begin{aligned}
& \mu_{(k)}^{\prime}=\frac{(n-1)^{(k-1)}(\alpha+1)^{[k-1]}}{(\alpha+\gamma+1)^{[k-1]}} \times \\
& {\left[\mu_{1}^{\prime}(n-k+1, \alpha+k-1, \gamma)+(k-1)\right]}
\end{aligned}
$$

Using (3.4), the $k^{\text {th }}$ factorial moment is obtained as 


\section{SIZE-BIASED POLYA-EGGENBERGER DISTRIBUTION}

$$
\begin{aligned}
& \mu_{(k)}^{\prime}=\frac{(n-1)^{(k-1)}(\alpha+1)^{[k-1]}}{(\alpha+\gamma+1)^{[k-1]}} \times \\
& {\left[\frac{(n-k)(\alpha+k)}{(\alpha+\gamma+k)}+(k-1)\right]}
\end{aligned}
$$

and the first four factorial moments are

$$
\begin{gathered}
\mu_{1}^{\prime}=\frac{(n-1)(\alpha+1)}{(\alpha+\gamma+1)} \\
\mu_{(2)}^{\prime}=\frac{(n-1)(n-2)(\alpha+1)(\alpha+2)}{(\alpha+\gamma+1)(\alpha+\gamma+2)}+\frac{(n-1)(\alpha+1)}{(\alpha+\gamma+1)} \\
\mu_{(3)}^{\prime}=\frac{(n-1)(n-2)(n-3)(\alpha+1)(\alpha+2)(\alpha+3)}{(\alpha+\gamma+1)(\alpha+\gamma+2)(\alpha+\gamma+3)} \\
+\frac{2(n-1)(n-2)(\alpha+1)(\alpha+2)}{(\alpha+\gamma+1)(\alpha+\gamma+2)}
\end{gathered}
$$$$
\begin{aligned}
& P(x)= \\
& {\left[\begin{array}{l}
\frac{(n-1) \ldots(n-x+1)}{(x-1) !} \\
\times \frac{(\alpha+1)(\alpha+2) \ldots(\alpha+x-1) \gamma(\gamma+1) \ldots(\gamma+n-x-1)}{(\alpha+\gamma+1)(\alpha+\gamma+2) \ldots(\alpha+\gamma+n-1)}
\end{array}\right]}
\end{aligned}
$$

Taking the limit $\gamma \rightarrow \infty$ such that $\frac{\alpha}{\gamma}=\theta$ results in

$$
P(x)=\frac{(n-1) \ldots(n-x+1)}{(x-1) !} \frac{\theta^{x-1}}{(1+\theta)^{n-1}} .
$$

Proceeding to limit $n \rightarrow \infty$, such that $n \theta=\lambda$, the equation reduces to a size-biased Poisson distribution with parameter $\lambda$.

Estimation

Different estimation techniques are now put forth to estimate the parameters of the proposed model. The model (2.3) has three parameters $n, \alpha$ and $\gamma$. The parameter $n$ is known, whereas the remaining two parameters $\alpha$ and $\gamma$ must be estimated.

The Moment Method

Let $m_{1}^{\prime}, m_{2}^{\prime}$ be the sample moments (about origin) of a size-biased PolyaEggenberger distribution (2.3). The method of moments consists in comparing the sample moments with the population moments of the distribution. The two equations thus obtained are

$$
m_{1}^{\prime}=\frac{(n-1)(\alpha+1)}{(\alpha+\gamma+1)}
$$

and

$$
m_{2}^{\prime}=\frac{(n-1)(n-2)(\alpha+1)(\alpha+2)}{(\alpha+\gamma+1)(\alpha+\gamma+2)} .
$$

Theorem 4.1Proof

The PMF of proposed model (2.3) is

Dividing (5.2) by (5.1) gives:

$$
(t+1) \frac{m_{2}^{\prime}}{m_{1}^{\prime}}=n \alpha-2 \alpha+2 n-4
$$


HASSAN, BILAL \& IMTIYAZ

where

$$
\alpha+\gamma+1=t
$$

From equation (5.1),

$$
\alpha=\frac{m_{1}^{\prime} t+1-n}{n-1}
$$

and, from (5.3),

$$
\alpha=\frac{(t+1) \frac{m_{2}^{\prime}}{m_{1}^{\prime}}+4-2 n}{n-2} .
$$

Eliminating $\alpha$ between (5.5) and (5.6) results in:

$$
\frac{m_{1}^{\prime} t+1-n}{n-1}=\frac{(t+1) \frac{m_{2}^{\prime}}{m_{1}^{\prime}}+4-2 n}{n-2},
$$

which, after simplification, gives the value of $t$ as

$$
t=\frac{m_{2}^{\prime}(n-1)-n+1}{m_{1}^{\prime 2}(n-2)-n\left(m_{1}^{\prime}+m_{2}^{\prime}\right)-2 m_{1}^{\prime}+m_{2}^{\prime}} .
$$

Substituting the value of $t$ from this equation into (5.5), the value of $\alpha$ can be obtained, and after substituting the values of $t$ and $\alpha$ into (5.4) the value of $\gamma$ can be obtained.

Using the Mean and First Two Cell Frequencies

Taking $x=1,2$ in the size-biased Polya-Eggenberger distribution (2.3) and equating these probabilities with their corresponding relative frequencies $\frac{f_{1}}{N}, \frac{f_{2}}{N}$ results in:

$$
\frac{\gamma^{[n-1]}}{(\alpha+\gamma+1)^{[n-1]}}=\frac{f_{1}}{N}
$$

and

$$
\frac{(\alpha+1) \gamma^{[n-2]}}{(\alpha+\gamma+1)^{[n-1]}}=\frac{f_{2}}{N}
$$

where

$$
N=\sum f_{i} .
$$

Dividing (5.8) by (5.7) the ratio

$$
\frac{f_{2}}{f_{1}}=\frac{(n-1)(\alpha+1)}{(\gamma+n-2)}
$$

is obtained. Eliminating $\alpha$ between (5.9) and (5.1), the value of $\gamma$ results as

$$
\gamma=\frac{(n-\bar{x}-1)(n-2)\left(f_{1}+f_{2}\right)}{\bar{x} f_{2}+(n-1)\left(\bar{x} f_{1}-f_{2}\right)} .
$$

Substituting the value of $\gamma$ from this equation into (5.1) the value of $\alpha$ can be obtained.

The Method of Maximum-Likelihood

The $\log$ likelihood function of sizebiased Polya-Eggenberger distribution is given by

$$
\begin{aligned}
\log L= & N \log \Gamma(n)-\sum f_{x} \log \Gamma(n-x+1) \\
& -\sum f_{x} \log \Gamma(x+1)+\sum f_{x} \log \Gamma(\alpha+x) \\
& -N \log \Gamma(\alpha+1)+\sum f_{x} \log \Gamma(\gamma+n-x) \\
& -N \log \Gamma(\gamma)+N \log \Gamma(\alpha+\gamma+1) \\
& -\sum f_{x} \log \Gamma(\alpha+\gamma+n),
\end{aligned}
$$

where $f_{x}$ is the observed frequency for the variate value $\mathrm{x}$ and $N=\sum f_{x}$.

The proposed model has two unknown parameters, namely, $(\alpha, \gamma)$.The $\log$ likelihood equations for estimating $\alpha$ and $\gamma$ are

$$
\frac{\partial \log L}{\partial \alpha}=0=-N \sum_{k=1}^{n-1} \frac{1}{\alpha+\gamma+k}+\sum f_{x} \sum_{k=1}^{x-1} \frac{1}{\alpha+k}
$$

and 


$$
\frac{\partial \log L}{\partial \gamma}=0=\sum f_{x} \sum_{k=0}^{n-x-1} \frac{1}{\gamma+k}+\sum f_{x} \sum_{k=1}^{n-1} \frac{1}{\alpha+\gamma+k}
$$

These equations do not provide direct solutions, thus an iterative solution method, such as Newton-Rampson or Fisher's scoring method, are required to solve these equations. The following system of equations may also be solved:

$$
\left(\widehat{\theta}-\theta_{0}\right)\left[\frac{\partial^{2} \log L}{\partial \theta^{2}}\right]_{\theta_{0}}=\left[\frac{-\partial \log L}{\partial \theta}\right]_{\boldsymbol{\theta}_{0}}
$$

where $\hat{\theta}=(\alpha, \gamma)$ is a parameter vector, the $\mathrm{ML}$ estimate of $\theta$ and $\theta_{0}$ is the trial value of $\theta$ which may first be obtained by equating the theoretical frequencies with the observed frequencies.

\section{Acknowledgement}

This project was supported by the Research Center, College of Science, King Saud University, Riyadh, Saudi Arabia.

\section{References}

Bosch, A. J. (1963). The Polya distribution. Satistica Neerlandica, 17, 201-213.

Eggenberger, F., \& Pólya, G. (1923).Uber die Statistik verketteter Vorgänge. Zeitschrift $\beta r$ Angewandte Mathematik und Mechanik (ZAMM), 3, 279-289.
Fisher, R. A. (1934). The effects of methods of ascertainment upon the estimation of frequencies. Annals of Eugenics, 6, 13-25.

Holgate, P. (1970). The modality of compound Poisson distribution. Biometrika, 57, 665-667.

Mahfoud, M., \& Patil, G. P. (1982).On weighted distributions. In Statistics and probability: Essays in honour of C. R. Rao, G. Kallianpur, P. Krishnaiah \& J. Ghosh (Eds.), 479-492. New York, NY: North-Holland.

Mishra, A., \& Sing, S. K. (1993).A sizebiazed Lagrangian Poisson distribution and its use in zero-truncated cases. Ranchi University Mathematical Journal, 24, 21-28.

Patil, G. K. (1981). Studies in statistical ecology involving weighted distributions. In Applications and new directions, proceedings of Indian statistical institute golden jubilee, J. K. Ghosh \& J. Roy (Eds), 478-503. Calcutta, India: Statistical Publishing Society.

Patil, G. K., \& Ord, J. K. (1976).On size-biased sampling and related form-invariant distributions.Sankhya, Series B, 38(1), 48-61.

Polya, G. (1930). Sur quelques points de la theorie des probabilities. Annals de l'Institut H. Poincare, 1, 117-161.

Rao, C. R. (1965). On discrete distributions arising out of methods of ascertainment. In Classical and contagious discrete distributions, G. P. Patil (Ed.), 320-332. Calcutta, India: Statistical Publishing Society.

Srodka, T. (1963).On approximation of Hypergeometric distributions. Zeszyty Naukowe Politechniki Lodzkiej, 10(53), 5-17. 